\title{
Various methods for isolating DNA from fruiting bodies on the example of Pleurotus pulmonarius (higher basidiomycetes) of the Novosibirsk Region for barcoding of edible and medicinal mushrooms
}

\author{
Vyacheslav Vlasenko*, Sergey Asbaganov and Anastasia Vlasenko \\ Central Siberian Botanical Garden SB RAS, 630090 Zolotodolinskaya st., 101, Novosibirsk, Russia
}

\begin{abstract}
We conducted experiments to increase the concentration of extracted DNA from the fruiting bodies of this species using various lysis buffers and extraction time. In general, DNA isolation according to the protocol using SDS made it possible to obtain higher concentrations thereof, as in the case of increasing the extraction time from 1 to 24 hours and using additional purification with chloroform.
\end{abstract}

\section{Introduction}

P. pulmonarius (Fig. 1) have medicinal properties, the polysaccharides contained in them exhibit a high antioxidant, antitumor, immunomodulatory, antiviral effects [1,2].

DNA barcoding is a potent approach for rapid identification of fungal specimens, it can be used to accurately identify edible and medicinal mushrooms.

In this study, experiments were conducted on the isolation of DNA from the fruit bodies of oyster mushrooms to obtain its highest concentration.

\section{Materials and methods}

Fruiting bodies of fungi were collected in the field studies in various habitats of the Novosibirsk Region. Dried specimens were preserved in the herbarium, they were used for molecular genetic studies.

An overview of taxa used for studies, shows the species names, herbarium voucher, habitats and substrates given in Table 1 .

DNA extraction and sequencing

Specimens of $P$. pulmonarius was used for molecular analysis. A fragment of fungal fruiting bodies $(20 \mathrm{mg})$ was homogenized in lysis buffer and extract the DNA with NucleoSpin ${ }^{\circledR}$ Plant II kit was used. The ITS1-5.8S-ITS2 region of the rDNA was amplified by PCR with the primers ITS1F and ITS4B (Table 2).

\footnotetext{
*Corresponding author: vlasenkomyces@mail.ru
} 

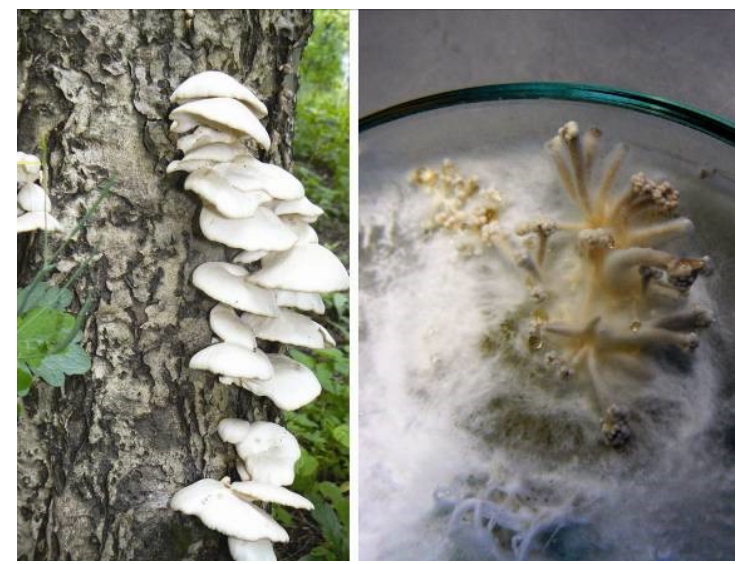

Fig. 1. Pleurotus pulmonarius - fruiting bodies in nature on Sorbus sibirica and mycelium in culture on agar medium in Petri dish.

Table 1. Pleurotoid fungi used for studies

\begin{tabular}{|l|c|c|c|}
\hline Species & Herbarium voucher & Habitat & Substrate \\
\hline Pleurotus pulmonarius & NSK 1014214 & birch forest & Sorbus sibirica \\
\hline Pleurotus pulmonarius & NSK 1014215 & birch forest & Betula pendula \\
\hline
\end{tabular}

Table 2. Primers used for studies

\begin{tabular}{|l|c|c|c|}
\hline Loci & Primers & (F/R) & Sequence $\left(5^{\prime}-3^{\prime}\right)$ \\
\hline ITS1-5.8S-ITS2 & ITS1-F & $\mathrm{F}$ & CTTGGTCATTTAGAGGAAGTAA \\
\hline ITS1-5.8S-ITS2 & ITS4-B & $\mathrm{R}$ & CAGGAGACTTGTACACGGTCCAG \\
\hline
\end{tabular}

For PCR, HS Taq DNA Polymerase (Evrogen, Russia) was used. PCR reactions were performed in a C1000 Thermal Cycler (Bio-Rad, USA). PCR results were checked at Gel Doc XR+ Imager (Bio-Rad, USA). DNA amplicons sequencing performed in SB RAS Genomics Core Facilities (Novosibirsk, Russia).

Sequences were aligned using ClustalW methods. The ITS sequences were aligned in MEGA 7. Phylogeny reconstruction was inferred using the UPGMA method. The percentage of replicate trees in which the associated taxa clustered together in the bootstrap test (100 replicates) is shown to be next to the branches. The evolutionary distances were computed using the Maximum Composite Likelihood method and were in the units of the number of base substitutions per site. The differences in the composition bias among sequences were considered in evolutionary comparisons. All ambiguous positions were removed for each sequence pair (pairwise deletion option). There were a total of 609 positions in the final dataset. Evolutionary analyses were conducted in MEGA 7.

\section{Results and Discussion}

Lysis buffers PL-1 (CTAB method) and PL-2 (SDS- method) were used. Two types of extraction were used: 1 hour according to the manufacturer's protocol and 24 hours with additional purification with chloroform. The results of measurements of the concentration of extracted DNA are presented in Table 3.

Table 3. The concentration of DNA isolated from the studied samples

\begin{tabular}{|c|c|c|c|c|}
\hline Samples & 1 & 2 & 1 & 2 \\
\hline Method & CTAB & CTAB & SDS & SDS \\
\hline C (extraction $1 \mathrm{~h})$ & 24 & 27 & 29 & 39 \\
\hline
\end{tabular}




\begin{tabular}{|l|l|l|l|l|}
$\mathrm{C}($ extraction $24 \mathrm{~h})$ & 172 & 94 & 38 & 106 \\
\hline
\end{tabular}

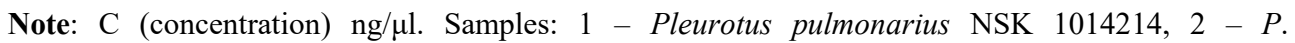
pulmonarius NSK 1014215. Optical density ratios A260/A280 = 1.84-1.97.

The results of PCR imaging are presented in Fig. 2.

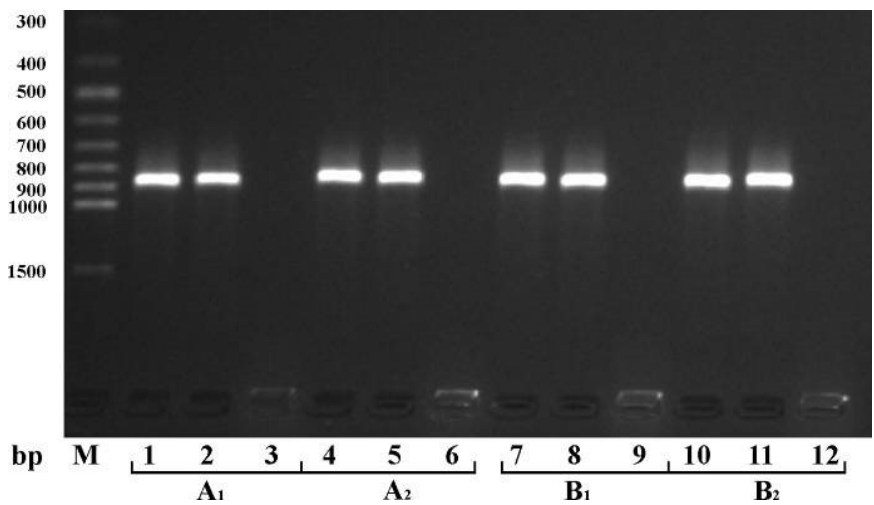

Fig. 2. Visualization of $P C R$ results. $C T A B$ - method: A (samples 1-6). A1 - manufacturer protocol extraction, A2 - extraction with an increase in extraction time. SDS - method: B (samples 7-12). B1 manufacturer protocol extraction B2 - extraction with an increase in extraction time. Samples DNA: 1, 4, 7, 10 - Pleurotus pulmonarius NSK 1014214. Samples DNA: 2, 4, 8, 11 - Pleurotus pulmonarius NSK 1014215. Samples DNA: 3, 6, 9, 12 -Meriderma carestiae myxomycete DNA was used for fungal-specific primer monitoring).

Phylogenetic analyses

Additional 2 ITS sequences of other Pleurotus species based on BLAST results and 4 ITS sequences of other species were retrieved from GenBank (http://www.ncbi.nlm.nih.gov/Genbank/). We first generated a 2 new sequence for the ITS1-5.8S-ITS2 region for $P$. pulmonarius. The final dataset consisted of 7 ITS sequences. An overview of all taxa and on sequences used for tree reconstruction, shows the species names, herbarium vouchers/strain and Genbank accession numbers given in Table 4.

Table 4. Sequences used in alignment

\begin{tabular}{|l|c|c|}
\hline Species & Herbarium voucher/Strain & Genbank accession numbers \\
\hline Pleurotus pulmonarius & NSK 1014214 & MN179418 \\
\hline Pleurotus pulmonarius & NSK 1014215 & MN179421 \\
\hline Pleurotus pulmonarius & FPPMK-L & JX429930 \\
\hline Pleurotus 'sajor-caju' & H-1 & JQ837470 \\
\hline Pleurotus cf. eryngii & C1 & FJ514549 \\
\hline Pleurotus ostreatus & 6689 & AY450345 \\
\hline Pleurotus populinus & 9936 & AY450346 \\
\hline
\end{tabular}

The molecular phylogenetic analyses placed the specimens of Pleurotus genus from Novosibirsk Region close to P. pulmonarius (Fig. 3). 


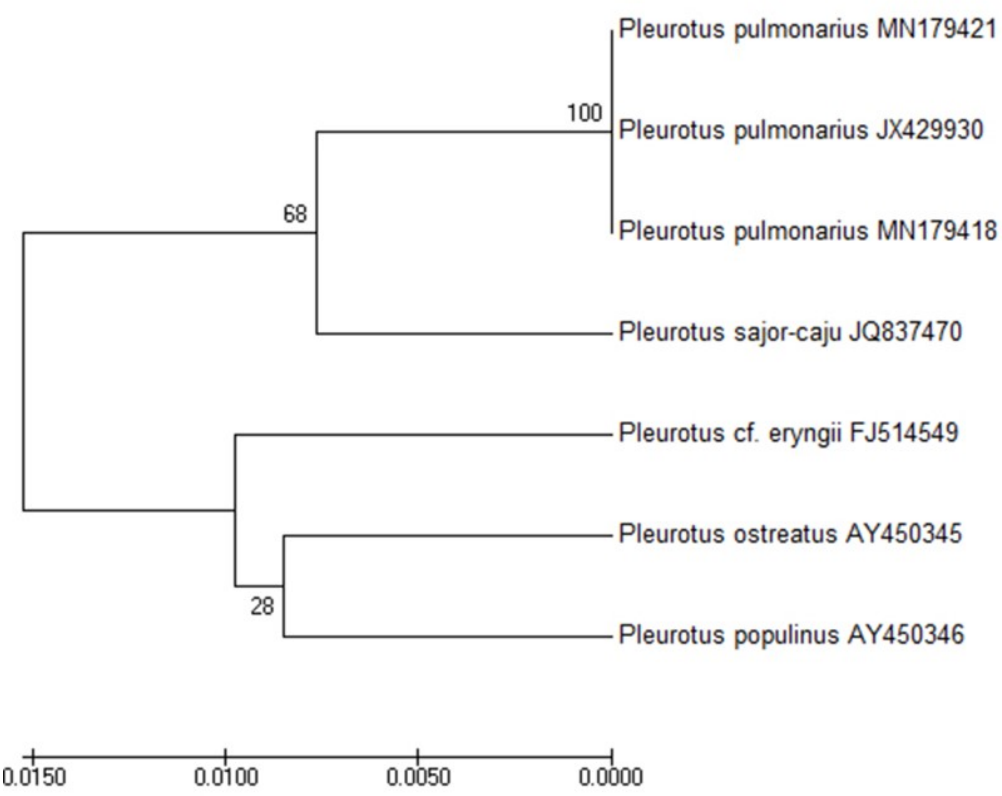

Fig. 3. UPGMA trees based on ITS sequences showing the phylogenetic relationships between the $P$. pulmonarius species from Novosibirsk Region and other related species of Pleurotus genus. Herbarium specimens from the Bioresources scientific collection (USU 440537) MG Popov Herbarium (NSK) were used.

\section{Conclusions}

DNA isolation according to the protocol using SDS allows to obtain higher concentrations, as and in the case of increasing the extraction time from 1 to 24 hours and using additional purification with chloroform.

\section{Acknowledgements}

The reported research was funded by Russian Foundation for Basic Research and the government of the Novosibirsk Region of the Russian Federation, grant № 18-44-543018 R_Mol_a.

\section{References}

1. T.V. Teplyakova, N.V. Psurtseva, T.A. Kosogova, N.A. Mazurkova, V.A. Khanin, V.A. Vlasenko. Int. J. Med. Mushr., 14(1) (2012)

2. V.A. Vlasenko, A.V. Vlasenko. BIO Web Conf., IV, 00044, 11 (2018) 